\title{
The Polymerase Chain Reaction in an Anemic Mode: How to Avoid Cold Oligodeoxyribonuclear Fusion
}

\author{
Kary B. Mullis \\ La Jolla, California 92037 and Specialty Laboratories, Inc., Santa Monica, \\ California 90404
}

here is a very simple relationship between the number of molecules of polymerase in a PCR and the number of primed templates that can be extended in a given cycle of that reaction. The ratio is one to one. None of the conventions currently in use for describing the quantitative aspects of biochemical reactions are very useful in illuminating such stoichiometrical relationships. Before turning to the spectre of cold oligodeoxyribonuclear fusion reactions, we would like to discuss this situation and propose a minor change.

Enzymes have been traded and generally utilized by the activity unit for about 100 years, a unit of DNA polymerase, for example, being the amount required to convert under certain conditions 10 nmoles of deoxyribonucleotides into an acidinsoluble form in $30 \mathrm{~min}$ : not a very useful definition nor one that conveys any useful information to the molecular biologist except price. Oligodeoxyribonucleotides are traded by the $\mathrm{OD}_{260}$ unit, which was defined out of necessity as that amount of an oligomer that when dissolved in $1 \mathrm{ml}$ will show an optical density of 1.0 at $260 \mathrm{~nm}$ across a $1-\mathrm{cm}$ pathlength. Other nucleic acids are described by weight, even when their molecular weights are known; and magnesium, buffers, and dNTPs mysteriously are described by the fractional part that their count represents of Avogadro's number, even though this may be a minuscule fraction. Although all these units can be converted to a common currency, they do not do so in one's mind automatically, and consequently a great deal of clarity is sacrificed in our communications with each other and with our own experimental results.

Perhaps the worst of the lot in terms of the number of errors it causes in day-to-day calculations is the system based on Avogadro's number. For here we must deal with infinitesimal fractions of preposterous amounts. We do not buy gasoline by the cubic mile for obvious reasons. Avogadro's number is not an intrinsic chemical constant nor is it suitable for biochemistry in the twentieth century. It is derived from a measurement of the size of the Earth heroically made by the French in the eighteenth century. The number depends on the Gram which came from the Cubic Centimeter full of water at $4^{\circ} \mathrm{C}$, which derived from the Meter, which had been defined as one ten-millionth of the distance from the North Pole to the Equator along a line passing between the Notre-Dame and the guy selling chestnuts there in the winter of 1799 . We should not feel obliged to use Avogadro's number unless it is really useful.

In this paper we will try not talking about things to the twenty-third power, and particularly will try to avoid saying things like "One haploid human cell should contain $1.5 \times 10^{-24}$ moles of any unique sequence." If there was any sense at all in installing the gram-molecular-weight of something as a unit of measure, then it was expressly to avoid the need for dealing with huge exponents, and was not introduced as providing a cryptic way of expressing the concept "one."

We will refer to molecules as if they were things, one or more at a time; if we need to refer to concentration, we will say how many molecules there are in a given volume. And since volumes in biochemistry are conveniently measured in microliters, we will use the microliter. This system has the advantage of clarity.

Thus, the amount of something, be it a protein, a nucleic acid, or a magnesium atom, will be described in terms of how many of them there are. And while we are simplifying, we will drop the annoying convention of superscripts and just say 4E12, for instance, if we mean we have 4 times 10 to the 12th power of something. Neither will we lapse into Latin text unless begged by the specific occasion. So one thing is 1 , two things are 2, a thousand is E3. There are approximately E4 copies of the human genome in a microliter of human blood. This concentration will be written $\mathrm{U} 4$, meaning ten to the fourth per microliter. Millimolar, which we will want to dispense with directly as a remnant of an ancient measuring system, we will for the time being translate as $6 \mathrm{U} 14$. This requires that we drop the .023 , but we don't know anyone who pipettes that well anyhow.

One of our PCR protocols looks like this: Note that the two columns are not describing concentrations that are truly equal; we have modified the actual concentrations slightly so as to 
conform more roundly to the new system. It is our experience that small changes in parameters like concentration in PCR do not matter.

$\begin{array}{lll} & \text { After } & \text { Before } \\ \text { Tris } \mathrm{HCl} & 50 \mathrm{U} 15 & 80 \mathrm{~mm} \\ \text { Ammonium sulfate } & 10 \mathrm{U} 15 & 20 \mathrm{~mm} \\ \text { Magnesium chloride } & 5 \mathrm{U} 15 & 10 \mathrm{~mm} \\ \text { dNTPs } & 5 \mathrm{U} 14 & 1 \mathrm{~mm} \\ \text { Oligonucleotides } & 5 \mathrm{U} 11 & 1 \mu \mathrm{M} \\ \text { Taq polymerase } & 3 \mathrm{U} 9 & 5 \mathrm{units} \\ \text { DNA, human } & 3 \mathrm{U} 3 & 1 \mu \mathrm{g}\end{array}$

Volume $100 \mu \mathrm{l}$

Cycles: alternately $65^{\circ} \mathrm{C}, 30 \mathrm{sec} ; 98^{\circ} \mathrm{C}$, $30 \mathrm{sec}$.

\section{THE POLYMERASE CHAIN REACTION}

In a PCR where we are trying to double the number of molecules representing a particular target sequence every cycle, we need enough oligonucleotides to prime all the appropriate sequences, and to do it before these rehybridize to themselves; and we need enough enzyme to engage each primed sequence; and then enough dNTPs to furnish the raw materials of polymerization. The enzyme, being the reagent dispensed by biochemical supply houses in the lowest concentration (3U10 to 3U11) almost always becomes limiting before any of the others. Enzyme limitation leads to something we call "anemic cycles." More will be said later of these and of the attendant cold deoxyribonuclear fusion reactions that they promote.

A typical PCR might begin with $3 E 5$ copies of human DNA and the alleged purpose of amplifying a single-copy human gene. In an indisputable ethidium bromide gel band, there are about 5E12 base pairs of DNA. That means, by way of an example, that for a 100-bp amplicon (I didn't make up this word; I heard it at a meeting but I like it) we need $5 E 11$ copies of the 100 bp amplicon in a $100-\mu 1$ reaction volume to have $5 \mathrm{E} 12$ base pairs in a $10-\mu l$ aliquot for the gel. So we need some number of cycles to amplify the $3 E 5$ copies to $5 \mathrm{E} 11$. How many?

A basic equation that describes PCR is an old one which describes a lot of things that grow by division:

$$
Y_{n}=(1+e)^{n}
$$

where $Y_{n}$ is the amplification factor after $n$ cycles at an efficiency of $e$, which is a number from 0 to 1 that refers to that fraction of sequences available for duplication in each cycle that actually got duplicated.

So how many cycles do we need? We take the natural logarithm and rearrange the formula:

$$
n=\ln Y_{n} / \ln (1+e)
$$

$Y_{n}$ in this case stands for the ratio of how many molecules we want to how many molecules we started with, and including the 10-fold aliquot factor we have

$$
Y_{n}=5 \mathrm{E} 11 / 3 \mathrm{E} 5=1.76 \mathrm{E} 6
$$

and if we say optimistically that $e=1$ then

$$
n=\ln 1.76 \mathrm{E} 6 / \ln 2=20.7 \text { cycles. }
$$

So in 21 cycles we should be able to start with 3E5 copies of human DNA and get $a$ band on an ethidium bromide gel from a one-tenth aliquot. Sometimes we do and sometimes we don't.

In the last cycle, assuming everything has worked perfectly, we would need to be making 2.5E11 molecules, which requires $2.5 \mathrm{E} 11$ molecules of Taq translating back to about 10 units. This requirement is independent and in spite of the specific activity of the enzyme. The specific activity is measured as initial velocity of polymerization on a long template such as M13; and what we are dealing with in PCR is not simply that, but rather the ability to synthesize many separate molecules. And here we must take into account, we think, the strong affinity which the thermophilic polymerases have for their substrates. Thus, the polymerases are disinclined to ever come loose from a DNA molecule, even when they have reached the end of it. Coming to the end of a template probably has no evolutionary precedence for them because the process of repair and replication in bacteria never involves the, perhaps shocking and at least novel, experience for a polymerase of reaching the end of a template. Bacterial genomes are round, and bacterial polymerases are unlikely to be any better equipped to jump off the end of a linear PCR amplicon than are tightrope walkers to walk off the end of a rope. (If this is certainly true then the denaturation step in PCR serves a double purpose, but this is another concept best left for now.) What is true is that when the number of primed templates exceeds the number of Taq molecules, some of the primed templates do not get extended. This is what we refer to as an anemic cycle.

Anemia will occur at an earlier or later cycle in our reaction depending on how many enzyme molecules we have employed and, very critically, on how many nontarget amplicons we have inadvertently created, and are amplifying along with the intended target. Nontarget amplicons can arise from a number of factors, but most frequently they are generated by one or more extension cycles being carried out under nonstringent priming conditions, i.e., low temperature.

If when anemia occurs the intended target is a predominant species in the reaction, then we can simply do extra cycles until we slowly build up the amount of target amplicon desired. For the amplification of human genes and especially of less complex targets, this is often close to being the case, which is why PCR generally works without a hitch for genomic targets, even though a considerable part of the amplification occurs under anemic conditions.

This is not the case, however, if one is trying to amplify something like a single molecule of a foreign DNA in the presence of 3E5 copies of a complex host DNA, like, for example, HIV from $30 \mu \mathrm{l}$ of human blood. Here the problem arises that when the reaction goes anemic, there are in the reaction mixture the requisite $3 \mathrm{E} 10$ fragments of primed DNA per unit of Taq, which is one molecular of primed DNA per molecule of Taq, and which is why the reaction is becoming anemic, but hardly any of these molecules correspond to the intended target. If, as is typical of most PCR protocols, anemia begins around cycle 15 , the target, which has been copied with $100 \%$ efficiency up till now, is present in only 5E4 copies and at the very low reaction efficiencies that rapidly occur within a few cycles of the appearance of anemia; the target is not likely to ever reach 5E11 copies and easy visibility on ethidium bromide gels. In fact, with 30 additional anemic cycles at yields around $20 \%$, the target may be amplified up 
another

$$
Y_{n}=(1.2)^{30}=237 \text {-fold }
$$

to $1.2 \mathrm{E} 7$ copies, where it might be detected with ${ }^{32} \mathrm{P}$ or recent methods employing enzyme linkage.

But it is also possible that amplification of the target might come to an almost complete halt due to the fact that: Once the state of anemia is reached not all amplicons will be subject to the same degree of further amplification, and it may not even be amplified 237-fold and may not be detected at all. For in anemia there is a shortage of resources in the reaction and only those fragments that compete best for them will be duplicated. The factors that govern this competition are not complicated and weigh heavily on the side of short fragments, and with this we arrive at the spectre of cold deoxyribonuclear fusion and the short ugly fragments to which it gives rise.

\section{COLD DEOXYRIBONUCLEAR FUSION}

From empirical observations no one will doubt that, once established, shorter fragments in an anemic reaction will be amplified preferentially. Little molecules seeming to cluster in the region on the gel that would be about two primers long if primers were double stranded seem to pop up out of nowhere and dominate the amplification. Something seems to be catalyzing the fusion of oligodeoxyribonucleotides. More cycles lead to more intensity of these little fragments but not much more of your target fragment. Their size has earned them the misnomer of "primer dimers," in spite of the fact that sequences have always revealed non-primer-derived core regions, which the concept of "dimer" does not imply. A bold solution to these "ugly little fragments" in the case that they do not interfere with the detection of one's intended amplicon was publicly announced by Richard Gibbs (in a talk at the Keystone UCLA Symposium on PCR in 1989). "Run them off the bottom of the gel."

As uplifting as this solution might have been to the people who have suffered from the "ugly little fragments," it is of no help in those cases where the appearance of these PCR artifacts interferes seriously with the amplification and detection of the intended target. What we are addressing here is the difference between the amplification of a genomic target (say $3 \mathrm{E} 5$ up to $5 \mathrm{E} 11$, or a little more than a million-fold) where the background amplicons are a nuisance that can be run off the gel and the amplification of a needle in a haystack (one copy up to 5E11 or 100billion-fold) where the nonintended background amplifications can prevent the intended target from ever showing up on the gel.

The more complex the background and the longer the period when robust amplification is occurring, the more likely will be the occurrence of a very efficiently amplified short fragment. When the reaction becomes anemic, this fragment will be selectively amplified further. It is a molecular scale model of evolution (especially since medium-sized amplicons as they accumulate can increasingly give rise to shorter amplicons by internal misprimings). Notice that both primers are required, rather than two copies of one. The amplicon produced from two copies of one primer would have internal hairpin symmetry in each single strand and would be amplified at a very low efficiency.

Theoretical considerations indicate that the inverse size dependence of amplification efficiency is a fairly steep one. The question becomes, then, in a practical sense, how to prevent the initial generation of these unwanted little fragments in what was hopefully to be a high-gain amplification of a usefully large fragment. Although it should be noted that when there is a choice in regard to the size of the intended amplicon, it certainly makes no sense, ceteris paribus, to design it larger than necessary.

One way to prevent short ugly fragments from overwhelming a reaction is to prevent the extension reactions from beginning until the temperature is high enough to insure that very few nonperfect-match primings get extended. The generation of very short fragments is inversely dependent on the stringency of priming and extension.

When a reaction mixture similar to that described earlier using heatdenatured human DNA was subjected to $5 \mathrm{~min}$ at room temperature, about 1 ng of new DNA was produced. This was not in any way associated with target amplification, as the primers were designed to amplify a sequence from the gag region of HIV and do not specifically amplify any human sequence. This was scattered background primer extension that occurred from the human DNA, and was detected as precipitable counts or a broad smear on a gel centered around $500 \mathrm{bp}$ when one of the dNTPs was $\alpha$-labeled. Somewhat surprisingly, this initial $5 \mathrm{~min}$, during which only $0.1 \%$ of new DNA was synthesized, had a devastating effect on subsequent amplification efficiency compared to a reaction where no primer extensions were allowed to proceed below $65^{\circ} \mathrm{C}$.

The powerful effect of the newly synthesized nanogram of DNA is due to the fact that each molecule begins with one of the primers. For purposes of the following discussion, we will describe these fragments as "halfamplicons." Now the probability of producing new DNA comprising full amplicons by two cycles of random priming, where in the second cycle priming on a half-amplicon generates a fragment with two perfect primer sites, will be given by an expression involving the square of the probability for producing half-amplicons. Thus, the probability for generating unwanted PCR amplicon DNA by repeating the 5min, low-stringency cycle to a first approximation would be

$P \times P=(1 \mathrm{ng} / 1 \mu \mathrm{g}) \times(1 \mathrm{ng} / 1 \mu \mathrm{g})=\mathrm{E}-6$

Assuming that the full amplicons would on average be half the size of the half-amplicons, the number of amplicons generated would be

$$
\begin{gathered}
N=(\mathrm{E}-6)(1.8 \mathrm{E} 15) / 250 \\
N=7.2 \mathrm{E} 6
\end{gathered}
$$

where $1.8 \mathrm{E} 15$ is the number of nucleotides in $1 \mu \mathrm{g}$ of DNA.

But in our experiment after subjecting the reaction mixture to one lowstringency cycle for $5 \mathrm{~min}$, we entered a normal PCR cycle where extension took place at $65^{\circ} \mathrm{C}$. We do not know the actual amount of new amplicon DNA that was synthesized in the second cycle, but it is instructive, in terms of understanding the influence that one low-temperature cycle can have on a PCR reaction, to examine a range of 
probabilities and examine the outcome for each. Below we have compared the number of amplicons of average size 250 nucleotides that would be produced in two cycles, where in the one case, $\mathrm{A}$, both cycles were done under a stringency that would produce new DNA with a probability of $P$, and where in the other case, $B$, the first cycle was done under low-stringency conditions in which new DNA was produced with a probability $\mathrm{E}-3$. The first two columns list the number of amplicons produced and the second two columns predict the number of cycles that will be required for those amplicons to give rise to an anemic reaction.

\begin{tabular}{|c|c|c|c|c|}
\hline \multicolumn{5}{|c|}{$\begin{array}{l}\text { AMPLICONS GENERATED AT CYCLE } \\
\text { TWO UNDER CONDITIONS OF } \\
\text { INCREASING STRINGENCY IN CASES } \\
\text { A AND B }\end{array}$} \\
\hline $\boldsymbol{P}$ & $\boldsymbol{A}$ & $\boldsymbol{B}$ & $\boldsymbol{A}$ & $\boldsymbol{B}$ \\
\hline$E-4$ & $1.4 \mathrm{E} 5$ & $1.4 \mathrm{E} 6$ & 21 & 17 \\
\hline$E-5$ & $1.4 \mathrm{E} 3$ & $1.4 \mathrm{E} 5$ & 27 & 21 \\
\hline$E-6$ & 14 & $1.4 \mathrm{E} 4$ & 34 & 24 \\
\hline$E-7$ & .14 & $1.4 \mathrm{E} 3$ & 41 & 27 \\
\hline$E-8$ & 0 & 140 & & 30 \\
\hline$E-9$ & 0 & 14 & & 34 \\
\hline
\end{tabular}

In case $\mathrm{B}$ corresponding to an amplification in which a cold cycle was performed, either intentionally or inadvertently, even if a very stringent routine were followed after the first cold cycle ( $E-8$, for instance), it would be very unlikely if a single target molecule could be amplified and readily detected in the presence of over 100 other amplicons. In this case, the reaction would be anemic after a minimum of only 30 more cycles, at which point a single molecule would have been amplified up to about 4E9, which would not yet be visible on an ethidium gel.

The statistical manipulations here have been very much first approximations, but the conclusions drawn are in accord with experimental results. In the experiment referred to above (K. Mullis, F. Ferre, and F. Faloona, in prep.), we were able to achieve the required sensitivity to detect one molecule of HIV DNA in the presence of $1 \mu \mathrm{g}$ (3E5 copies) of human DNA on an ethidium-stained gel. This represents a 1000 -fold increase in sensitivity compared to the same protocol after a 5-min exposure of the complete reaction mixture to room temperature. Starting the reaction cold with what we considered to be native DNA lowered the detection limits about 100 -fold under our conditions. It is our opinion, but we have not demonstrated this, that thorough denaturation of chromosomally integrated targets is necessary to insure quantitatively reproducible amplifications, especially when the number of starting sequences is close to one. This requires boiling for several minutes and cannot be done in the presence of enzyme. The simplest way to avoid cold oligodeoxyribonuclear fusion and its consequent ugly little fragments is to put the reaction together with the DNA denatured, but without the dNTPs, until the temperature is brought up to about $80^{\circ} \mathrm{C}$. At this point, with the tubes in a heat block ready to go into a machine that is holding at $80^{\circ} \mathrm{C}$, the dNTPs can be added to a reactionally inactive mixture, taken up to $98^{\circ} \mathrm{C}$ for $30 \mathrm{sec}$, and then all samples will enter the PCR cycles equivalently; and uniformity and high-gain amplifications can be expected and accomplished.

An awareness of the nature of anemia in PCRs, its potentially catastrophic results, and ways of preventing it, only one of which has been mentioned here, might be beneficial to many workers who have experienced problems with PCR. Two other examples that come to mind where an abundance of unwanted amplicons might lead to a failure of the desired amplification are amplification from RNA where a low-stringency reverse transcriptase step is interjected and experiments in the clever but as yet to be extensively applied technique of inverse PCR. 


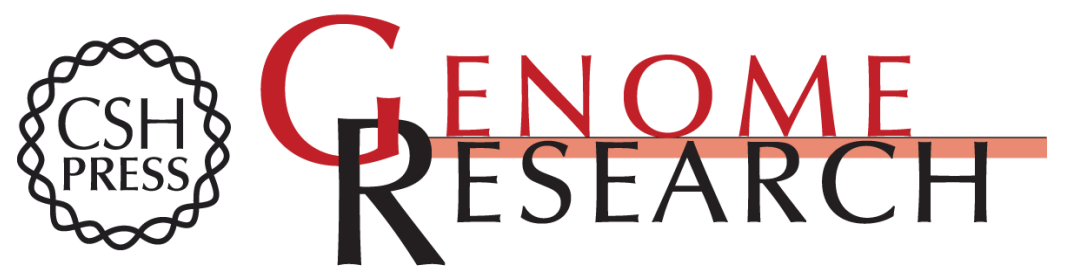

\section{The Polymerase Chain Reaction in an Anemic Mode: How to Avoid Cold Oligodeoxyribonuclear Fusion}

Kary B. Mullis

Genome Res. 1991 1: 1-4

Access the most recent version at doi:10.1101/gr.1.1.1

\section{License}

Email Alerting Service

Receive free email alerts when new articles cite this article - sign up in the box at the top right corner of the article or click here.

\section{Affordable, Accurate} Sequencing.

To subscribe to Genome Research go to: https://genome.cshlp.org/subscriptions 